\title{
Severe traumatic tricuspid insufficiency detected 10 years after blunt chest
} trauma

Emmert, M Y ; Prêtre, René ; Sündermann, S ; Weber, B ; Bettex, D A ; Hoerstrup, S P ; Falk, V

DOI: https://doi.org/10.1007/s00392-010-0219-6

Posted at the Zurich Open Repository and Archive, University of Zurich ZORA URL: https://doi.org/10.5167/uzh-36143

Journal Article

Published Version

Originally published at:

Emmert, M Y; Prêtre, René; Sündermann, S; Weber, B; Bettex, D A; Hoerstrup, S P; Falk, V (2011). Severe traumatic tricuspid insufficiency detected 10 years after blunt chest trauma. Clinical Research in Cardiology, 100(2):177-179.

DOI: https://doi.org/10.1007/s00392-010-0219-6 


\title{
Severe traumatic tricuspid insufficiency detected 10 years after blunt chest trauma
}

\author{
M. Y. Emmert $\cdot$ R. Pretre $\cdot$ S. Suendermann · \\ B. Weber $\cdot$ D. A. Bettex $\cdot$ S. P. Hoerstrup $\cdot$ \\ V. Falk
}

Received: 7 June 2010/Accepted: 25 August 2010/Published online: 15 September 2010

(C) Springer-Verlag 2010

\section{Sirs:}

A 29 years old male patient was found to have a systolic murmur on a routine medical examination. He was referred to a cardiologist for trans-thoracic echocardiography (TTE), which revealed a severe insufficiency of the tricuspid valve with a flail anterior leaflet due to complete rupture of a papillary muscle (Carpentier's Type II dysfunction). The right ventricle was hypertrophic and dilated with a normal systolic function. There were no signs of pulmonary hypertension and the left ventricular ejection fraction was preserved $(\mathrm{EF}=54 \%)$. The patient did not have any cardiovascular history such as myocardial ischemia, rheumatic fever or infective endocarditis. On the other hand, he mentioned to have had two severe car accidents 11 and 10 years ago. In detail, he suffered from two blunt, frontal chest traumas including multiple costal fractures and a clavicle fracture. Therefore, a traumatic injury of the tricuspid valve was highly suspected. In the further history, the patient denied any cardiac symptoms such as syncope, dyspnea, angina pectoris or palpitations, but reported occasional apical chest pains. The patient was referred to our tertiary center and was scheduled for surgical repair of the tricuspid valve. An intraoperative trans-esophageal echocardiography (TOE) confirmed the preoperative findings (Fig. 1a, b). The operation was performed via classical sternotomy under cardiopulmonary

M. Y. Emmert $(\bowtie) \cdot$ R. Pretre $\cdot$ S. Suendermann $\cdot$ B. Weber . S. P. Hoerstrup · V. Falk

Clinic for Cardiovascular Surgery, University Hospital Zurich, Raemi Street 100, 8091 Zurich, Switzerland

e-mail: maximilian_emmert@web.de

D. A. Bettex

Institute of Anaesthesiology, University Hospital Zurich,

Zurich, Switzerland bypass with bicaval cannulation. After opening the right atrium, the rupture of the papillary muscle was confirmed and the anterior leaflet was massively extended. The anterior leaflet was re-fixed using six Gore-Tex sutures as artificial chordae $(6 \times$ Gore-Tex 6-0 sutures) and was stabilized with a rigid annuloplasty ring (Physio $34 \mathrm{~mm}$, Edwards Lifesciences, Irvine, USA). The intraoperative TOE revealed a successful repair of the valve without detectable insufficiency (Fig. 2a, b). The rest of the procedure was uneventful and the patient was referred to the ICU. After 1 day in the ICU and 5 more days in the normal ward, the patient was discharged and made a swift recovery. Three months after surgery, the patient was in good condition with no residual tricuspid regurgitation.

We report a rare case of a severe tricuspid valve regurgitation following blunt chest trauma, which has remained asymptomatic and undetected for over 10 years.

Posttraumatic tricuspid valve insufficiency is a rare occurrence, but due to its anterior location, it remains the most frequently reported valve injury following blunt chest trauma [1]. Major mechanisms for traumatic tricuspid valve injury include rupture of the papillary muscles, chordal disruption, free rupture of the valve leaflets or complete destruction of the valve [2].

Although there have been many advances in echocardiography, allowing a faster diagnosis and resulting in an earlier and more effective treatment, the diagnosis of traumatic tricuspid valve insufficiency is often delayed or even can be completely unrecognized,. Major reasons for this problem are the general rarity, the absence or inhomogeneity of symptoms and the presence of coexisting life-threatening injuries such as hemato-pneumothorax, which have to be treated with priority, obscuring other pathologies [3, 4]. For this reason, it is not exceptional for patients to present years after the trauma [5]. In our patient, 

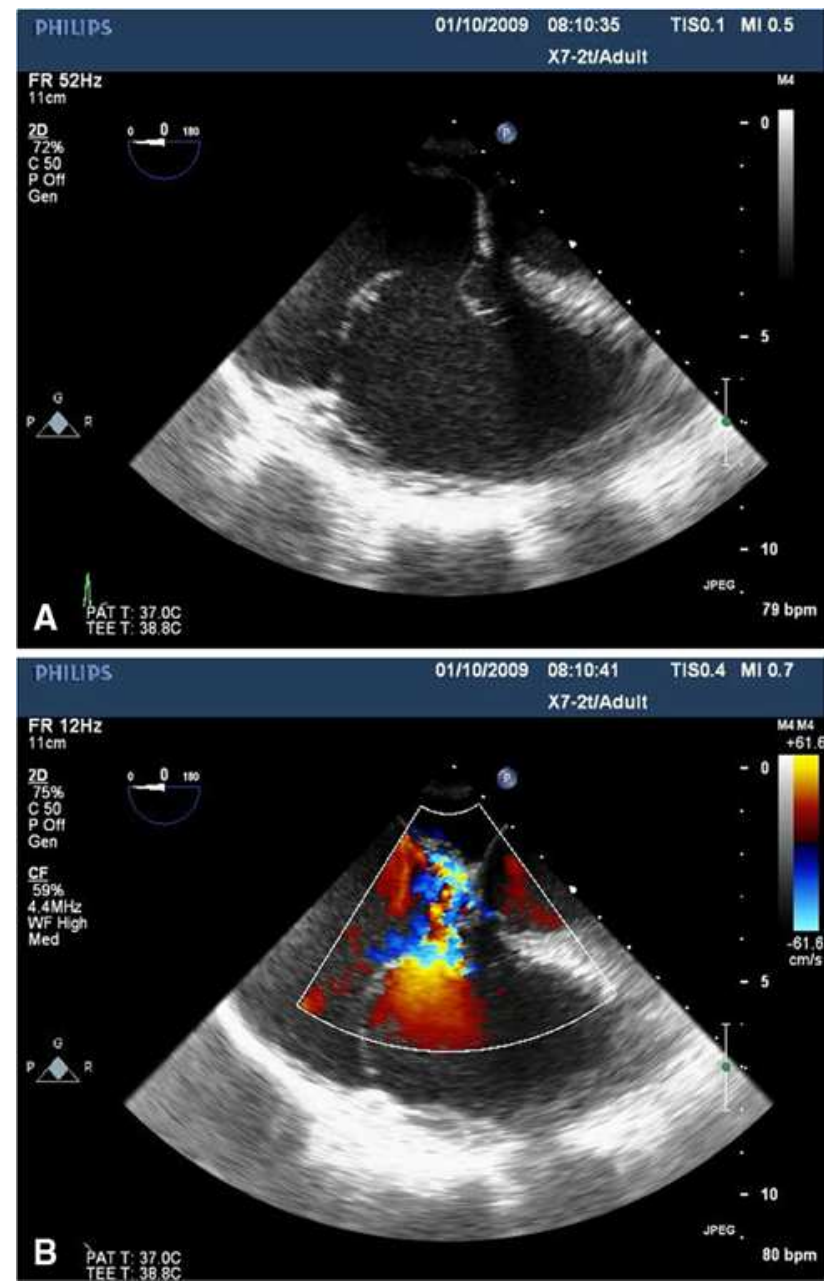

Fig. 1 a,b Intraoperative trans-esophageal echocardiography (TOE) confirmed the preoperative findings and revealed a severe insufficiency of the tricuspid valve with a flail anterior leaflet due to a complete rupture of a papillary muscle

the diagnosis was delayed for more than 10 years as the patient was asymptomatic for the major part of the time and reported to have only unspecific and irregular pain in the apex area. In addition, the exact time of rupture remained unclear, since he had two car accidents. Even if he had a follow-up echocardiography after the first accident, it might be possible that he could have had an initial trauma of the valve without rupture, but with elongation or injury, and with the second trauma, or spontaneously, the rupture could have occurred.

The early symptoms usually reported are mainly fatigue, dyspnea on exertion and palpitations; sometimes, EKG signs of incomplete or complete right bundle branch block due to a progressive dilatation of the tricuspid annulus may be found. A progressive right heart dilatation, which may end up with right heart failure, has been described [4]. As seen in our case, patients can often tolerate acute right heart volume overload and reach a compensated status,
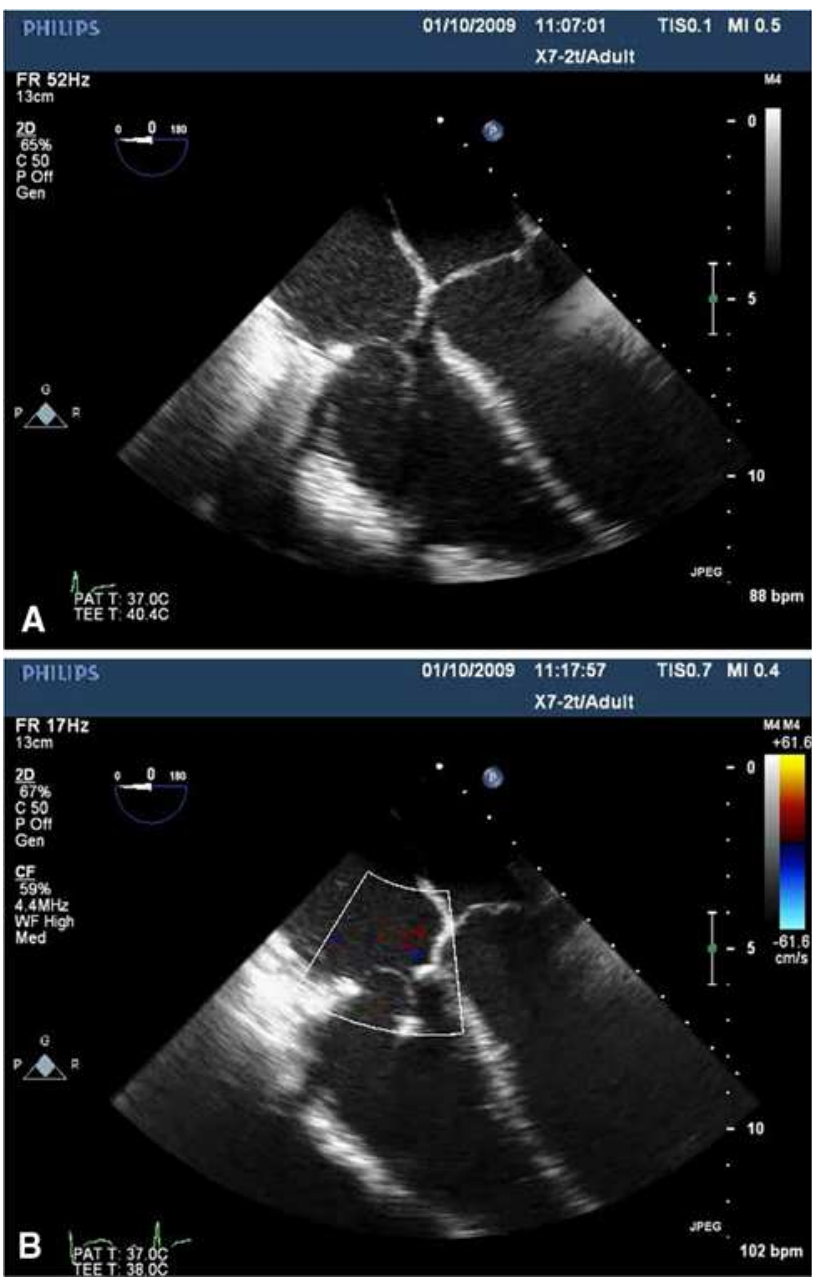

Fig. 2 a,b Intraoperative TOE displayed successful valve repair using six artificial chordae and a stabilizing annuloplasty ring

remaining asymptomatic for a long duration of time [4, 6]. However, to prevent progressive right ventricular dysfunction and preserve normal heart rhythm, early diagnosis and treatment are recommended and should be focused whenever possible [5].

The surgical strategy should be adapted individually to the patient's specific pathology. Besides the general consensus to repair the valve whenever feasible [7], various approaches and techniques for effective surgical treatment have been described. The utilization of synthetic materials to replace the ruptured chordae or papillary muscle has been reported to be a promising strategy. In our case, we used Gore-Tex sutures to re-fix the anterior leaflet followed by the implantation of a rigid annuloplasty ring to reduce and stabilize the annular size, respecting Carpentier's principles of valve repair.

This report should alert all involved clinicians to consider traumatic tricuspid valve injury whenever a patient presents with blunt chest trauma, even if they initially focus on acute injuries. Furthermore, trans-thoracic or 
trans-esophageal echocardiography is mandatory in this setting and, even if it is not possible in the situation of an acute trauma, it should be routinely planned as a follow-up screening.

\section{References}

1. Mattox KL, Feliciano DV, Burch J, Beall AC Jr, Jordan GL Jr, De Bakey ME (1989) Five thousand seven hundred sixty cardiovascular injuries in 4459 patients. Epidemiologic evolution 1958 to 1987. Ann Surg 2096:698-705 discussion 706-7

2. Noera G, Sanguinetti M, Pensa P et al (1991) Tricuspid valve incompetence caused by nonpenetrating thoracic trauma. Ann Thorac Surg 512:320-322
3. Asbach S, Siegenthaler MP, Beyersdorf F, Bode C, Geibel A (2006) Aortic valve rupture after blunt chest trauma. Clin Res Cardiol 9512:675-679

4. Schuster I, Graf S, Klaar U, Seitelberger R, Mundigler G, Binder T (2008) Heterogeneity of traumatic injury of the tricuspid valve: a report of four cases. Wien Klin Wochenschr 12015(16):499-503

5. van Son JA, Starr A (1995) Repair of traumatic tricuspid valve insufficiency by trabecular muscle elevation. Ann Thorac Surg 593:740-742

6. Luo GH, Ma WG, Sun HS, Xu JP, Sun LZ, Hu SS (2005) Correction of traumatic tricuspid insufficiency using the double orifice technique. Asian Cardiovasc Thorac Ann 133:238-240

7. Tang GH, David TE, Singh SK, Maganti MD, Armstrong S, Borger MA (2006) Tricuspid valve repair with an annuloplasty ring results in improved long-term outcomes. Circulation 1141(Suppl):I577-I581 